\title{
Quasistatic rheology, force transmission and fabric properties of a packing of irregular polyhedral particles
}

\author{
E. Azéma ${ }^{a, b}$, F. Radjai ${ }^{a}$, G. Saussine ${ }^{c}$ \\ ${ }^{a}$ Laboratoire de Mécanique et Génie Civil, Université Montpellier 2, Place Eugène \\ Bataillon, 34095 Montpellier cedex 05 \\ b Present address : Laboratoire Central des Ponts et Chaussées, Démarches \\ Durables en Génie Civil, 44341 Bouguenais cedex (Site de Nantes) \\ ${ }^{\mathrm{c}}$ Innovation and Research Departement of SNCF, 45 rue de Londres, 75379 Paris \\ Cedex 08
}

\begin{abstract}
By means of contact dynamics simulations, we investigate a dense packing composed of polyhedral particles under quasistatic shearing. The effect of particle shape is analyzed by comparing the polyhedra packing with a packing of similar characteristics except for the spherical shape of the particles. The polyhedra packing shows higher shear stress and dilatancy but similar stress-dilatancy relation compared to the sphere packing. A harmonic approximation of granular fabric is presented in terms of branch vectors (connecting particle centers) and contact force components along and perpendicular to the branch vectors. It is found that the origin of enhanced shear strength of the polyhedra packing lies in its higher force anisotropy with respect to the sphere packing which has a higher fabric anisotropy. Various contact types (face-vertex, face-face, etc) contribute differently to force transmission and fabric anisotropy. In particular, most face-face contacts belong to strong force chains along the major principal stress direction whereas vertex-face contacts are correlated with weak forces and oriented on average along the minor principal stress direction in steady shearing.
\end{abstract}

Key words: granular materials, polyhedral particles, contact dynamics method, shear strength, granular fabric, force chain PACS: 61.43.Bn, 81.05.Rm, 83.80.Fg, 45.70.Cc

Email addresses: emilien.azema@lcpc.fr (E. Azéma), radjai@lmgc.univ-montp2.fr (F. Radjai), gilles.saussine@sncf.fr (G. Saussine).

Preprint submitted to Mechanics of Material 25 October 2018 


\section{Introduction}

Many recent numerical studies of granular media deal with model systems composed of spherical particles. The use of simplified particle shapes and contact interactions is needed in order to focus on the collective behavior of particles which is at the origin of many specific properties of granular materials. On the other hand, the numerical treatment of complex particle shapes by discrete element methods was until very recently out of reach due to demanding computational resources. There is presently, however, considerable scope for the numerical investigation of complex granular packings. This is not only due to available computer power and memory but also because during more than two decades of intense research in this field, many fundamental aspects of granular media have already been established for simplified particle shapes. In particular, various microscopic features such as fabric anisotropy (Kruvt and Rothenburg 1996], Bathurst and Rothenburg [1988], Rothenburg and Bathurst 1989], Radiai et al. [1998], Kruyt and Rothenburg [2004]), force transmission (Liu et al. [1995], Radiai et al. [1996], Coppersmith et al. [1996], Mueth et al. [1998], Lovol et al. [1999], Bardenhagen et al. [2000], Antony [2001], Silbert et al. [2002], Metzger [2004], Maimudar and Behringer [2005]) and friction mobilization (Radjai et al. [1998], Staron and Radjai [2005]) have been analyzed for circular particles (in 2D) and spheres (in 3D). Hence, a recurrent issue today is how robust these findings are with respect to particle shape (Ouadfel and Rothenburg |2001|, Antonv and Kuhn |2004], Cambou et al. |2004|, Nouguier-Lehon et al. [2003], Alonso-Marroquin and Herrmann [2002], Pena et al. [2005, 2006a, b], Azéma et al. [2007]).

The issue of shape effect opens actually the door to a vast and substantial scientific domain given a multitude of potential particle morphologies. Several well-known examples are elongated and platy shapes (occurring in biomaterials and pharmaceutical applications), angular and facetted shapes (occurring in geomaterials) and nonconvex shapes (occurring in sintered powders). The macroscopic shear behavior is considerably influenced by particle shape. Rounded particles enhance flowability whereas angular shape is susceptible to improve shear strength, a factor of vital importance to civilengineering applications (Nouguier-Lehon et al. [2003]). In many engineering applications the particle shapes need to be optimized in order to increase performance (Markland [1981], Wu and Thompson [2000], Lim and MacDowel 2005], Saussine et al. [2006], Lobo-Guerrero and Vallejo [2006], Lu and McDowe] [2007]).

In this paper, we employ the contact dynamics method to investigate the slow shear behavior of granular media composed of polyhedral particles. The facetted shapes give rise to a rich microstructure where the particles touch at their faces, edges and vertices. Face-face contacts are expected to play a 
major role in force transmission and statics of polyhedra by accommodating long force chains that are basically unstable in a packing composed of spheres. In order to isolate the effects arising from particle shape, the data from the polyhedra packing will be compared with a packing of spherical particles that, apart from the particle shape, is identical in all respects (preparation, friction coefficients, particle size distribution) to the polyhedra packing. Both packings are subjected to monotonous triaxial compression.

The numerical procedures will be presented with a brief technical introduction to the detection and treatment of contacts between polyhedra in the framework of the contact dynamics method. We will consider the stress-strain and volume-change behavior. The harmonic approximation of the fabric and additive decomposition of the stress tensor into fabric and force anisotropies will be presented in detail. This will allow us to assess in clear terms the origins of shear strength in the polyhedra packing from fabric and force anisotropies in comparison to the sphere packing. The probability density functions of normal forces will be studied and compared between the two assemblies. Finally, we will focus on the contact networks of polyhedral particles and the role played by different contact categories with respect to force transmission.

\section{Numerical method}

In this section we briefly introduce the contact dynamics (CD) method with polyhedral particles and the numerical procedures used for sample preparation.

\subsection{Contact dynamics method with polyhedra}

The CD method is based on implicit time integration and nonsmooth formulation of mutual exclusion and dry friction between particles (Jean and Moreau [1992], Moreau [1994], Radjai and Roux [1999], Dubois and Jean [2003]). The equations of motion are formulated as differential inclusions in which velocity jumps replace accelerations (Moreau [1994]). The unilateral contact interactions and Coulomb friction law are represented as set-valued force laws. The implementation of the time-stepping scheme requires the geometrical description of each potential contact in terms of contact position and its normal unit vector.

At each time step, all kinematic constraints implied by enduring contacts are simultaneously taken into account together with the equations of motion in order to determine all velocities and contact forces in the system. This problem 
is solved by an iterative process pertaining to the non-linear Gauss-Seidel method that consists of solving a single contact problem, with other contact forces being treated as known, and iteratively updating the forces until a given convergence criterion is achieved. The method is thus able to deal properly with the nonlocal character of the momentum transfers resulting from the impenetrability of the particles and friction law.

The CD method is unconditionally stable due to its inherent implicit time integration method. The uniqueness of the solution at each time step is not guaranteed for perfectly rigid particles. However, by initializing each step with the forces calculated in the preceding step, the variability of admissible solutions shrinks to the numerical resolution. In the discrete element methods based on molecular dynamics, this "force history" is, by construction, included in the particle positions.

The treatment of a contact interaction between two particles requires the identification of the contact zone and a "common plane". For rigid particles it is possible to define this contact zone by a finite set of points. Before applying the contact detection algorithm between a pair of particles of irregular shapes, a "bounding box" method is used to compute a list of particle pairs potentially in contact. Then, for each pair, the first step is to determine if an overlap exists through a 3D extension of the "shadow overlap method" (Saussine [2004], Dubois and Jean [2003]). Several algorithms exist for overlap determination between convex polyhedra (Cundall and Strack [1979], Cundall [1988], Nezami et al. [2004, 2006], Dubois and Jean [2003], Saussine [2004], Saussine et al. [2006], Pérales [2007]). When an overlap occurs, the contact plane is determined by computing the intersection between the two particles.

The contacts between polyhedral particles belong to different categories, namely face-face, edge-face, vertex-face, edge-edge, vertex-vertex, vertex-edge; see Fig. 1. The vertex-vertex and vertex-edge contacts are practically absent. In all cases, we determine one, two or three contact points which provide a good description of the contact zone. In this paper, the vertex-edge and edge-edge contacts are referred to as "simple" contacts whereas the edge-face and faceface contacts are treated as "double" and "triple" contacts since their representation involves 2 and 3 distinct points on the common plane, respectively.

For our simulations, we used the LMGC90 which is a multipurpose software developed in Montpellier, capable of modeling a collection of deformable or undeformable particles of various shapes by different resolution algorithms (Dubois and Jean [2003]). 

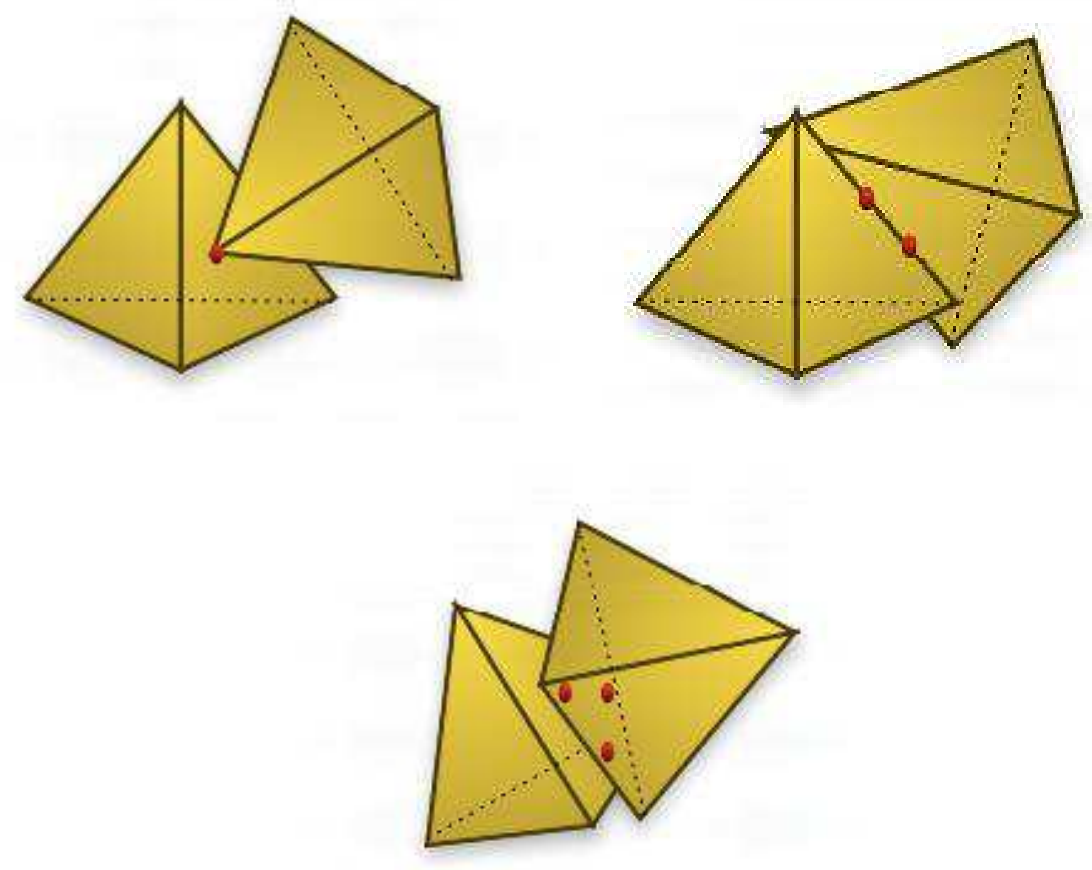

Fig. 1. Different types of contacts between two polyhedra.

\subsection{Sample preparation}

We generate two numerical samples. The first sample (S1) is composed of 36933 polyhedra. The particle shape are taken from a library of 1000 digitalised ballast grains provided by the French Railway Company SNCF. Each particle has at most 70 faces and 37 vertices and at least 12 faces and 8 vertices. Fig. 2 shows several examples of the polyhedral particles used in the simulations. The size of a particle is defined as two times the largest distance between the barycenter and the vertices of the particle, to which we will refer as "diameter" below. We used the following size distribution: $50 \%$ of diameter $d_{\min }=2.5$ $\mathrm{cm}, 34 \%$ of diameter $3.75 \mathrm{~cm}, 16 \%$ of diameter $d_{\max }=5 \mathrm{~cm}$. This distribution represents an approximation of that of railway ballast grains. The sample contains $7.110^{5}$ vertices and more than $10^{6}$ faces, the average numbers being 20 and 35, respectively. The second sample (S2) is composed of 19998 spheres with exactly the same size distribution as in S1. Fig. 3 shows a snapshot of the two samples in equilibrium state after deposition and isotropic compression under a constant stress of $\sigma_{0}=10^{4} \mathrm{~Pa}$ in a rectangular box at zero gravity.

The coefficient of friction is 0.5 between the particles in both samples and 0 with the walls. The normal and tangential coefficients of restitution are 0 . The zero restitution simplifies the deposition and compaction process by 


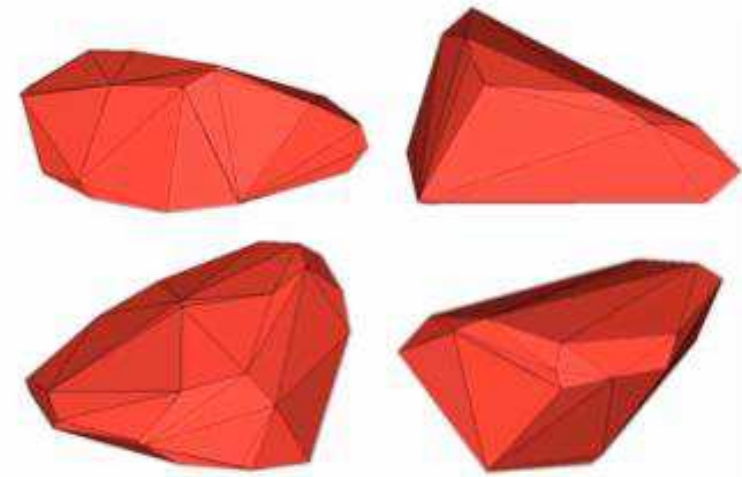

Fig. 2. Examples of polyhedra used in the simulations.
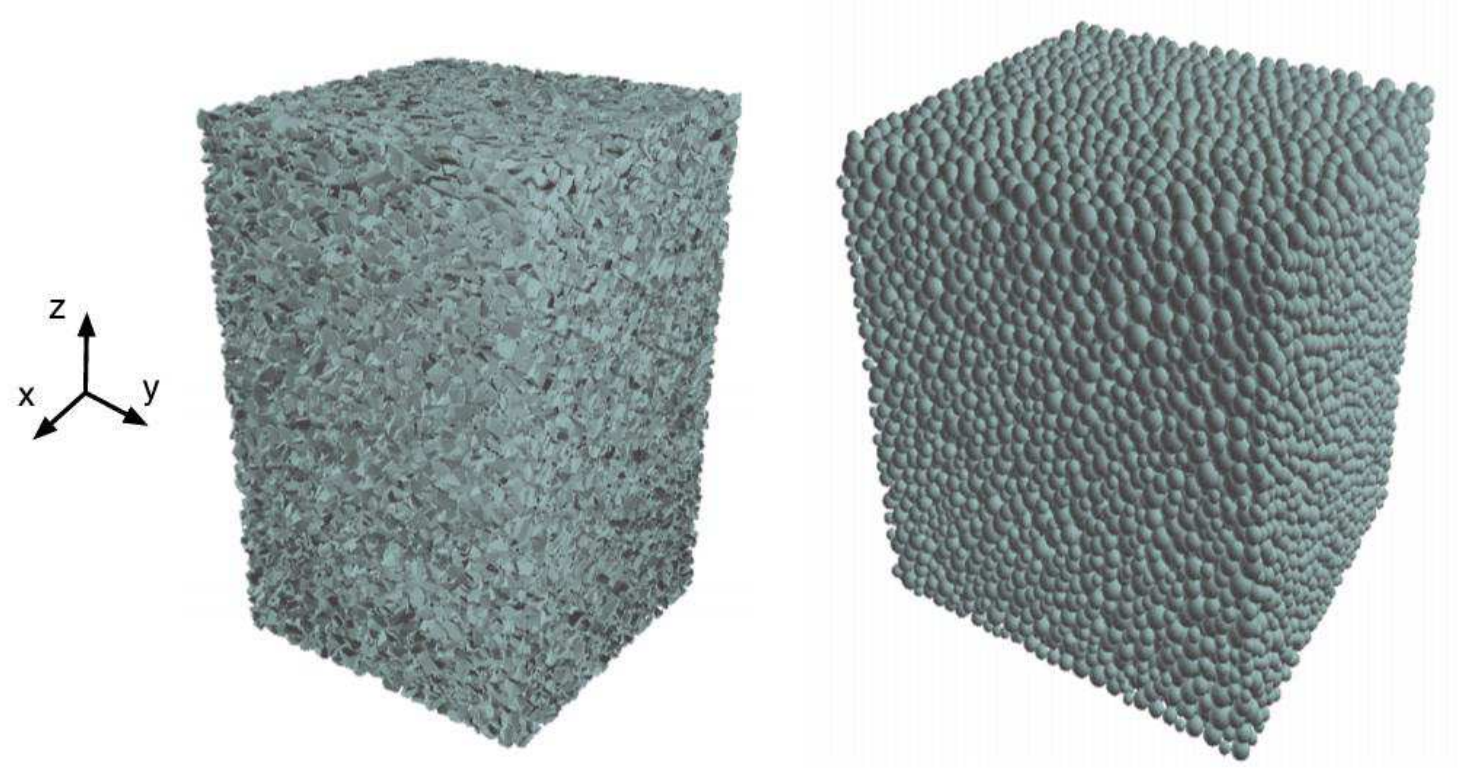

Fig. 3. Snapshots of the two packings S1 (polyhedra) and S2 (spheres). The walls are not shown

enhancing dissipation during dynamics rearrangements. The initial value of the solid fraction is $\rho \simeq 0.6$ in both samples. Both samples have a nearly square bottom of side such that $L \approx l$ and an aspect ratio $H / L \simeq 2$, where $H$ is the height. The initial configuration is defined by $H_{0} \simeq 30 D_{M}$ for S1 and for $\mathrm{S} 2$ with $D_{M}$ the mean diameter.

The isotropic samples are subjected to vertical compression by imposing a constant downward velocity of $10 \mathrm{~cm} / \mathrm{s}$ on the upper wall and a constant confining stress $\sigma_{2}=\sigma_{3}=\sigma_{0}$ on the lateral walls. Each simulation is stopped for a vertical deformation of $30 \%$. The time step was $2.10^{-4} \mathrm{~s}$. The CPU time was $210^{-3} \mathrm{~s}$ for $\mathrm{S} 1$ and $10^{-3} \mathrm{~s}$ for $\mathrm{S} 2$, per particle and per time step on an Apple G5 computer. The deformation process can be considered to be quasistatic in view of the weak kinetic energy injected into the samples 
compared to the static pressure. This can be expressed more generally through the inertial number defined as (GDR-MiDi [2004]):

$$
I=\dot{\varepsilon} \sqrt{\frac{m}{d p}}
$$

where $\dot{\varepsilon}=\dot{H} / H$ is the vertical strain rate, $m$ is the total mass, $p$ is the mean pressure and $d$ is the mean particle diameter. In our simulations, we have $I \simeq 10^{-3}$, corresponding to the quasistatic limit.

\section{Stress-strain behavior}

In this section, we compare the stress-strain and volume-change behavior between the packings of polyhedra (packing S1) and spheres (packing S2). The stress and strain variables are defined from numerical data. For the estimation of the stress tensor, we use the "tensorial moment" $\boldsymbol{M}^{i}$ of each particle i defined by (Moreau [1997], Staron and Radjai [2005]):

$$
M_{\alpha \beta}^{i}=\sum_{c \in i} f_{\alpha}^{c} r_{\beta}^{c}
$$

where $f_{\alpha}^{c}$ is the $\alpha$ component of the force exerted on particle i at the contact $\mathrm{c}, r_{\beta}^{c}$ is the $\beta$ component of the position vector of the same contact $\mathrm{c}$, and the summation runs over all contact neighbors of particle i (noted briefly by $c \in i)$.

It can be shown that the tensorial moment of a collection of rigid particles is the sum of the tensorial moments of individual particles (Moreau [1997]). The stress tensor $\boldsymbol{\sigma}$ for a packing of volume $V$ is simply given by (Moreau [1997], Staron and Radjai [2005]):

$$
\boldsymbol{\sigma}=\frac{1}{V} \sum_{i \in V} \boldsymbol{M}^{i}=\frac{1}{V} \sum_{c \in V} f_{\alpha}^{c} \ell_{\beta}^{c},
$$

where $\boldsymbol{\ell}^{c}$ is the branch vector joining the centers of the two touching particles at the contact $c$. Remark that the first summation runs over all particles whereas the second summation involves the contacts, each contact appearing only once.

Under triaxial conditions with vertical compression, we have $\sigma_{1} \geq \sigma_{2}=\sigma_{3}$, where the $\sigma_{\alpha}$ are the stress principal values. Using the Cambridge representa- 
tion, we define the mean stress $p$ and stress deviator $q$ by (Airey and Wood [1988]) :

$$
\begin{aligned}
p & =\frac{1}{3}\left(\sigma_{1}+\sigma_{2}+\sigma_{3}\right), \\
q & =\frac{1}{3}\left(\sigma_{1}-\sigma_{3}\right) .
\end{aligned}
$$

For our system of perfectly rigid particles, the stress state is characterized by the mean stress $p$ and the normalized shear stress $q / p$.

The cumulative strain components $\varepsilon_{\alpha}$ are defined by

$$
\begin{aligned}
& \varepsilon_{1}=\int_{H_{0}}^{H} \frac{d H^{\prime}}{H^{\prime}}=\ln \left(1+\frac{\Delta H}{H_{0}}\right), \\
& \varepsilon_{2}=\int_{L_{0}}^{L} \frac{d L^{\prime}}{L^{\prime}}=\ln \left(1+\frac{\Delta L}{L_{0}}\right), \\
& \varepsilon_{3}=\int_{l_{0}}^{l} \frac{d l^{\prime}}{l^{\prime}}=\ln \left(1+\frac{\Delta l}{l_{0}}\right),
\end{aligned}
$$

where $H_{0}, l_{0}$ and $L_{0}$ are the initial height, width and length of the simulation box, respectively and $\Delta H=H_{0}-H, \Delta l=l_{0}-l$ and $\Delta L=L_{0}-L$ are the corresponding cumulative displacements. The volumetric strain is given by

$$
\varepsilon_{p}=\int_{V_{0}}^{V} \frac{d V^{\prime}}{V^{\prime}}=\ln \left(1+\frac{\Delta V}{V_{0}}\right)
$$

where $V_{0}$ is the initial volume and $\Delta V=V-V_{0}$ is the total volume change. The cumulative shear strain is defined by

$$
\varepsilon_{q} \equiv \varepsilon_{1}-\varepsilon_{2}
$$

Figure 4 displays the evolution of $q / p$ for the packings S1 and S2 as a function of $\varepsilon_{q}$. For both packings, we observe a classical behavior characterized by a hardening behavior followed by (slight) softening and a stress plateau corresponding to the critical state of soil mechanics (Mitchell and Soga [2005]). The critical-state strength in the case of polyhedra $(\simeq 0.46)$ is twice as high as that of spheres $(\simeq 0.23)$. This implies that the polyhedra packing has a 


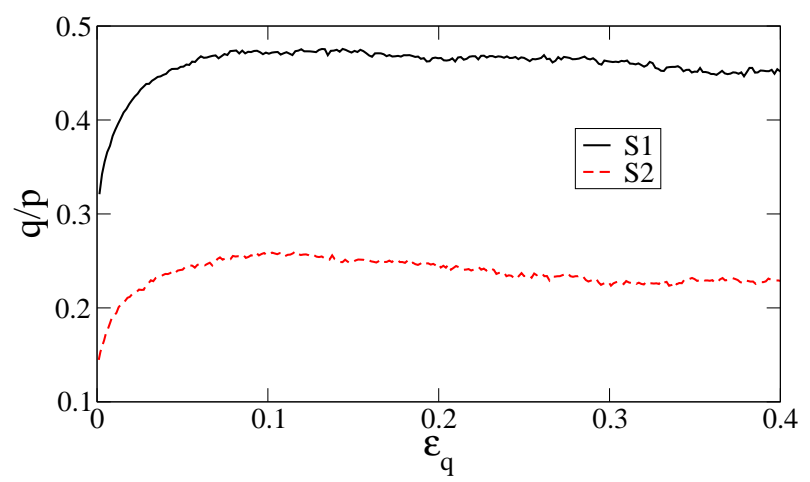

Fig. 4. The strength parameter $q / p$ as a function of shear strain $\varepsilon_{q}$ for the polyhedra packing S1 and sphere packing S2.

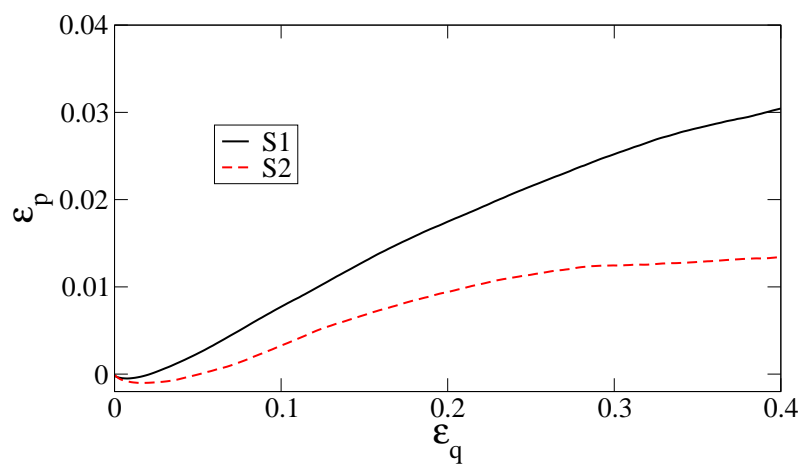

Fig. 5. The volume change $\varepsilon_{p}$ as a function of shear strain $\varepsilon_{q}$ for the packings $\mathrm{S} 1$ et S2.

higher angle of internal friction $\varphi$ defined by

$$
\sin \varphi=\frac{3 q}{2 p+q}
$$

At the critical state, we have $\varphi=\varphi_{0}=34^{\circ}$ for $\mathrm{S} 1$ and $\varphi_{0}=18^{\circ}$ for S2.

Figure 5 shows the volumetric strain $\varepsilon_{p}$ as a function of shear strain $\varepsilon_{q}$ in $\mathrm{S} 1$ and S2. In both packings, we observe an early compaction slightly larger in S2 than in S1. The subsequent dilation is lower in S2 and the critical state with isochoric deformation is reached at $\varepsilon_{q}=0.3$. Dilation in $\mathrm{S} 1$ continues with a decreasing rate of volume change but the isochoric plateau is not fully reached. The dilatancy can be expressed in terms of the dilation angle $\psi$ defined by

$$
\sin \psi=\frac{\varepsilon_{p}}{\varepsilon_{q}}
$$

We have $\psi \simeq 5^{\circ}$ for $\mathrm{S} 1$ and $\psi \simeq 2.5^{\circ}$ for $\mathrm{S} 2$ at the stress peak state. 


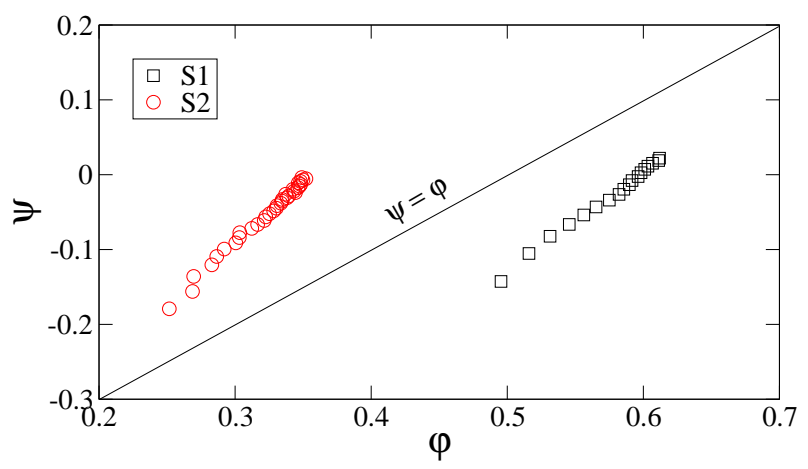

Fig. 6. The stress-dilatancy diagram representing the relation between the internal angle of friction and the dilation angle for polyhedra and spheres.

The variation of $\psi$ versus $\varphi$, a sort of stress-dilatancy diagram (Wood [1990]), is displayed in Fig. 6 for polyhedra and spheres. For both packings, we have

$$
\varphi \simeq k \psi+\varphi_{0}
$$

where $k$ is a constant slightly smaller than 1 in both packings. This correlation between dilatancy and shear stress during stress-strain transients is a consequence of energy balance. The mechanical work performed on the system is partially dissipated in contact interactions and partially used in volume change (Radjai and Roux [2004]). Several stress-dilatancy relations have been proposed as flow rules for plastic deformations of granular media (Wood [1990]). The relation (13) associates the peak state to the largest positive value of dilatancy and the critical state to zero dilatancy. It shows the "non associated" character of the flow rule in granular media (an associated flow rule implying $\varphi=\psi$ ).

\section{Harmonic representation of the fabric}

The expression of stress tensor in Eq. (3) is an arithmetic mean involving the branch vectors and contact forces. Hence, in order to analyze the shear strength properties of the polyhedra packing compared to the sphere packing, we need a statistical description of the granular microstructure (texture or fabric) and force transmission.

In the presence of steric exclusions, the granular microstructure is highly disordered at the particle scale (Troaded [2002], Troadec et al. [2002]). Since mechanical interactions are governed by contact and friction, the relevant descriptors of the microstructure are related to the contact network. At the lowest order, the contact network is characterized by the coordination number $z$ which describes the compactness of a packing. This is a crude scalar 


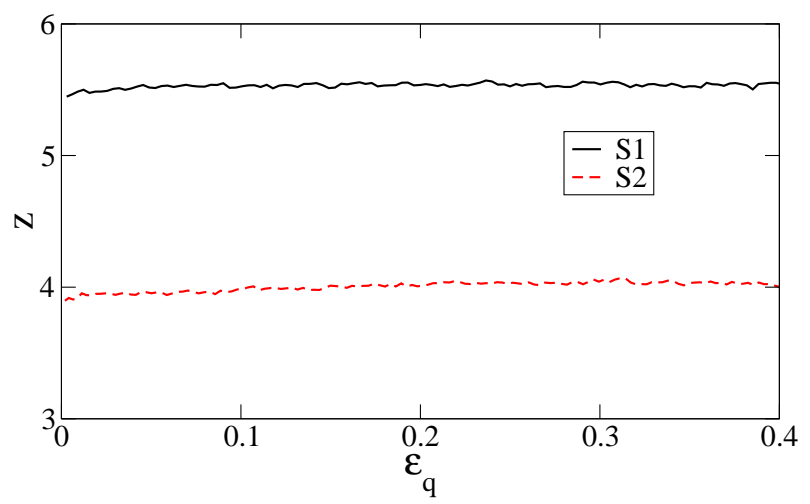

Fig. 7. Evolution of the coordination number $z$ as a function of the cumulative shear strain $\varepsilon_{q}$ for polyhedra (S1) and spheres (S2)

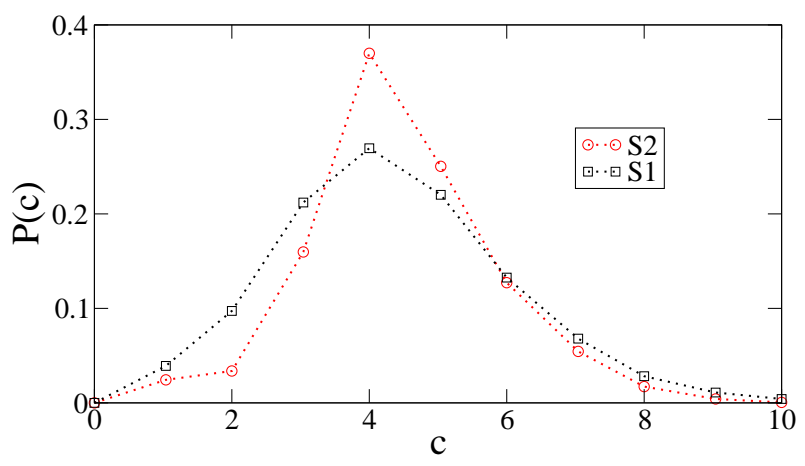

Fig. 8. The connectivity $P(c)$ of the contact network for the packings S1 and S2.

information in view of the complex arrangement of the particles, but it is well-known that the compactness, generally expressed in terms of the solid fraction, controls the stress-strain behavior under monotonous shearing. Let us remark here that double and triple contact types (see section 2) are counted as single contacts for the coordination number although they are represented by two and three contact points, respectively, in the numerical treatment of interactions between polyhedra.

The evolution of $z$ for polyhedra and spheres is shown in Fig. 7 as a function of $\varepsilon_{q}$. It is remarkable that $z$ is nearly constant in spite of the overall dilation in both packings. We have $z \simeq 5.5$ for polyhedra and $z \simeq 4$ for spheres. The connectivity of the contact network can be characterized in more detail by the fraction $P(c)$ of particles with exactly $c$ contact neighbors. The coordination number is the mean value of $c: z=\sum_{c} c P(c)$. Fig. 8 shows $P(c)$ for S1 and S2 in the critical state. The distribution is broader in S1 than in S2. This shows the wider range of potential equilibrium states in the polyhedra packing. For both packings, we observe a peak centered on $c=4$ with a higher probability for S2.

Since the shear stress corresponds to the deviation of stress components from the mean stress $p$ along different space directions, the coordination number 


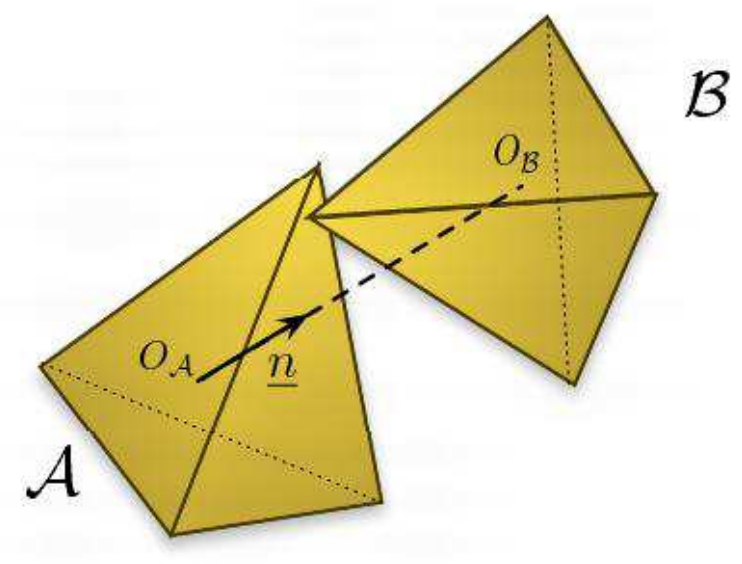

Fig. 9. Geometry of a contact between two polyhedra.

$z$ as a scalar quantity cannot account for the shear stress and its evolution with strain. Indeed, the expression of the stress tensor suggests that the useful information for the analysis of shear stress is the density and average force as a function of contact orientation. Such functions can be expanded in spherical harmonics in 3D (Ouadfel and Rothenburg [2001]).

Let $\boldsymbol{n}$ be the unit vector along the branch vector $\boldsymbol{\ell}$; Fig. 9. We set

$$
\ell=\ell n
$$

where $\ell$ is the length of the branch vector. We remark that the unit vector $\boldsymbol{n}$ does not coincide with the contact normal except in the case of spheres. We consider the components of the contact force in a local frame defined by $\boldsymbol{n}$ and an orthoradial unit vector $\boldsymbol{t}$ :

$$
\boldsymbol{f}=f_{n} \boldsymbol{n}+f_{t} \boldsymbol{t}
$$

where $f_{n}$ and $f_{t}$ are the radial and orthoradial components of the contact force, respectively. The writing of Eq. (15) assumes that $\boldsymbol{t}$ is oriented along the orthoradial force.

We now define the angular averages associated with the branch vectors $\boldsymbol{\ell}$ and contact force vectors $\boldsymbol{f}$. Let $\mathcal{A}(\Omega)$ be the set of branch vectors pointing in the direction $\Omega \equiv(\theta, \phi)$ up to a solid angle $d \Omega$ and $N_{c}(\Omega)$ its cardinal. The angles $\theta$ and $\phi$ are shown in Fig. 10. The angular averages are defined as follows:

$$
P_{\Omega}(\Omega)=\frac{N_{c}(\Omega)}{N_{c}}
$$




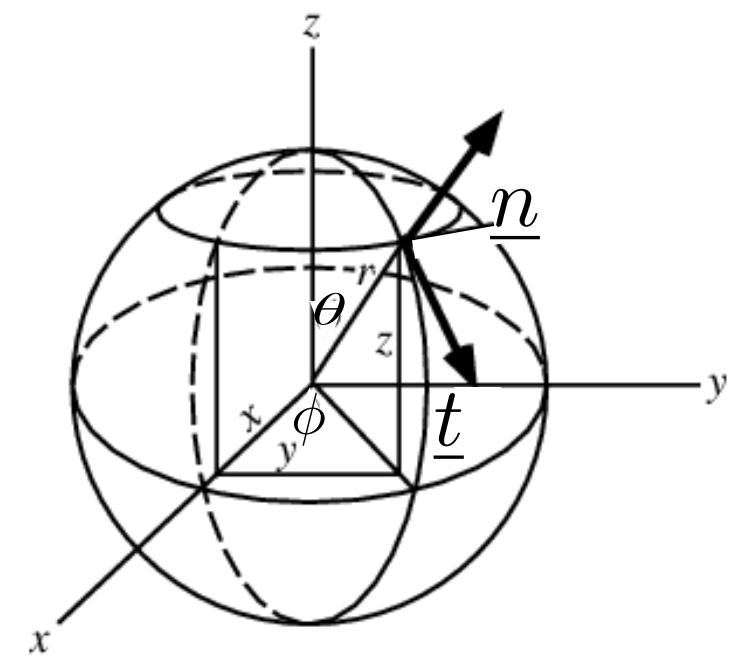

Fig. 10. Spherical coordinates.

$$
\begin{aligned}
\langle\ell\rangle(\Omega) & =\frac{1}{N_{c}(\Omega)} \sum_{c \in \mathcal{A}(\Omega)} \ell^{c}, \\
\left\langle f_{n}\right\rangle(\Omega) & =\frac{1}{N_{c}(\Omega)} \sum_{c \in \mathcal{A}(\Omega)} f_{n}^{c}, \\
\left\langle f_{t}\right\rangle(\Omega) & =\frac{1}{N_{c}(\Omega)} \sum_{c \in \mathcal{A}(\Omega)} f_{t}^{c},
\end{aligned}
$$

where $N_{c}=\int N_{c}(\Omega) d \Omega$ is the total number of contacts, and $\ell^{c}, f_{n}^{c}$, and $f_{t}^{c}$ are the actual values of branch vector length, radial force and orthoradial force for contact $c$, respectively.

Under the axisymmetric conditions of our simulations, the four functions defined in Eq. (19) are independent of $\phi$. Fig.11] displays a polar representation of these functions in the $\theta$-plane for polyhedra (S1) and spheres (S2) at $\varepsilon_{q}=0.3$. We observe an anisotropic behavior in all cases except in $\langle\ell\rangle(\theta)$ for S2. A weak anisotropy can be seen for S1 in the latter case. The peak values occur along the compression axis except for $\left\langle f_{t}\right\rangle(\theta)$ in which the peaks are inclined at $\pi / 4$ with respect to the vertical. The magnitude of anisotropy is larger for polyhedra compared to spheres except for $P_{\Omega}(\theta)$ which is weakly anisotropic for polyhedra.

The simple shapes of the above functions suggest that harmonic approximation based on spherical harmonics at leading terms captures their anisotropies. There are 9 second-order basis functions $Y_{m}^{l}(\theta, \phi)$. But only the functions compatible with the symmetries of the problem, namely independent with respect to $\phi$ and $\pi$-periodic as a function of $\theta$, are admissible. For $P_{\Omega}(\theta)$ as a scalar, and $\langle\ell\rangle(\theta)$ and $\left\langle f_{n}\right\rangle(\theta)$ as radial components of the vectors, the 

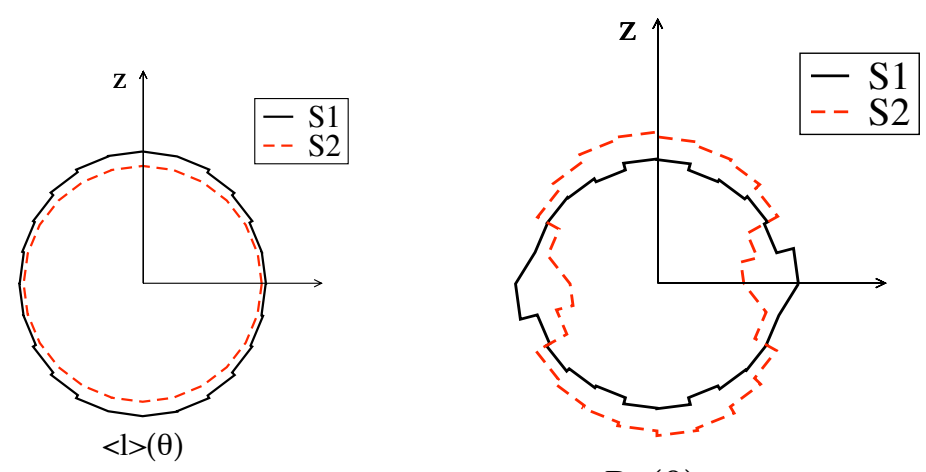

$$
\mathrm{P}_{\Omega}(\theta)
$$
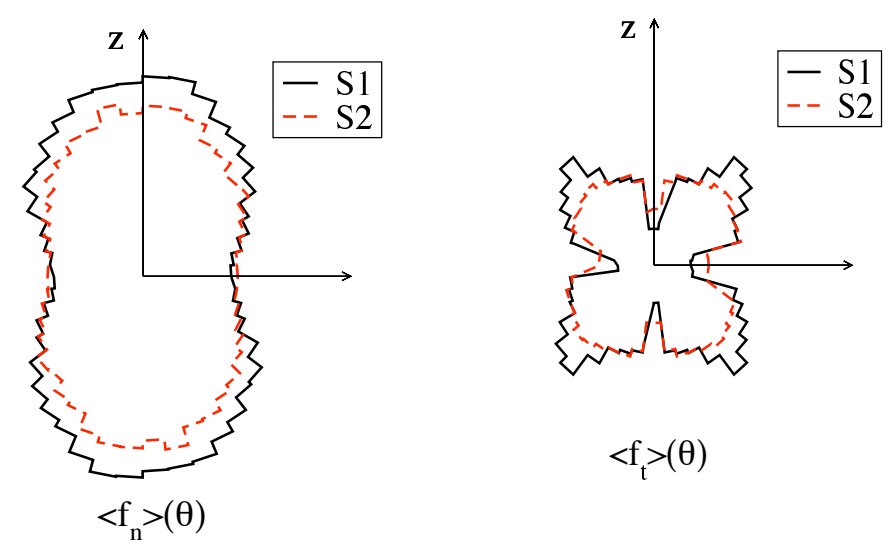

Fig. 11. Polar representation of density probability function $P_{\Omega}(\theta),\left\langle f_{n}\right\rangle(\theta),\left\langle f_{t}\right\rangle(\theta)$ and $\langle\ell\rangle(\theta)$ for $\mathrm{S} 1$ et $\mathrm{S} 2$ in residual state.

only admissible functions are $Y_{0}^{0}=1$ and $Y_{2}^{0}=3 \cos ^{2} \theta-1$. For $\left\langle f_{t}\right\rangle(\theta)$ as orthoradial component of a vector, the only function independent of $\phi$ and perpendicular to $Y_{0}^{0}=1$ and $Y_{2}^{0}=3 \cos ^{2} \theta-1$ is $\sin 2 \theta$. Hence, within the harmonic model of fabric and force, we have

$$
\begin{aligned}
P_{\Omega}(\theta) & =\frac{1}{4 \pi}\left\{1+a\left[3 \cos ^{2} \theta-1\right]\right\}, \\
\langle\ell\rangle(\theta) & =\ell_{0}\left\{1+a_{l}\left[3 \cos ^{2} \theta-1\right]\right\} \\
\left\langle f_{n}\right\rangle(\theta) & =f_{0}\left\{1+a_{n}\left[3 \cos ^{2} \theta-1\right]\right\}, \\
\left\langle f_{t}\right\rangle(\theta) & =f_{0} a_{t} \sin 2[\theta],
\end{aligned}
$$

where $a, a_{l}, a_{n}$ and $a_{t}$ are the anisotropy parameters, $\ell_{0}$ is the mean branch vector length, and $f_{0}$ the mean force. The probability density function $P_{\Omega}(\theta)$ is normalized to $1\left(\int_{\mathcal{S}} P_{\Omega}(\Omega) d \Omega=1\right.$, where $\mathcal{S}$ is a sphere of unit radius $)$. The values of the anisotropies $a, a_{l}, a_{n}$ and $a_{t}$ can be calculated from generalized 

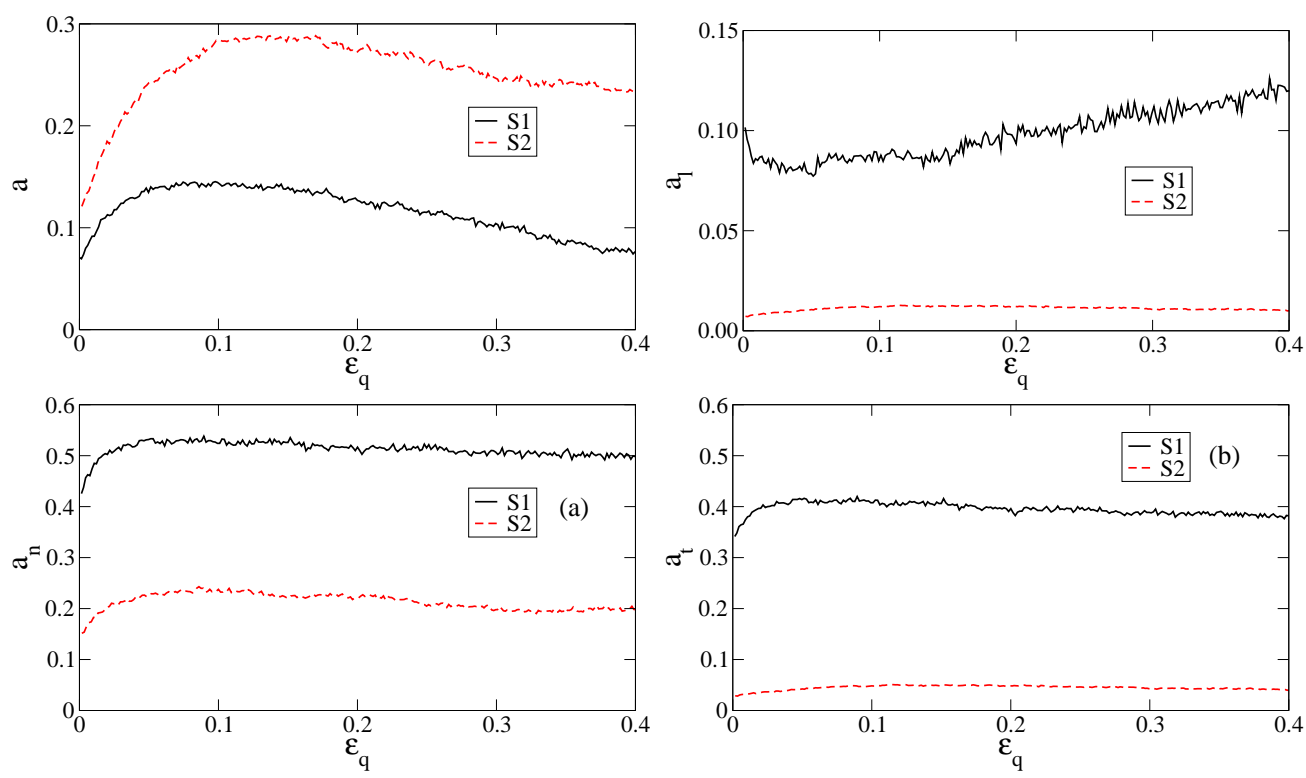

Fig. 12. Evolution of anisotropies $a, a_{l}, a_{n}$ and $a_{t}$ with $\varepsilon_{q}$ for packings S1 and S2.

fabric tensors introduced in Appendix A.

The evolution of the anisotropies with $\varepsilon_{q}$ are displayed in Fig. 12 for our packings $\mathrm{S} 1$ and $\mathrm{S} 2$. The fabric orientation anisotropy $a$ increases with $\varepsilon_{q}$ and relaxes to a plateau after passing by a pronounced peak. Its value is systematically larger for spheres than for polyhedra (by a factor 3 in the critical state). The branch vector anisotropy $a_{l}$ is quite low compared to other anisotropies and its value all along shearing is negligible for spheres. It is remarkable that $a_{l}$ for polyhedra declines (as $\varepsilon_{p}$, see Fig 5) at the beginning of shearing. The radial force anisotropy $a_{n}$ increases as the fabric anisotropy and tends to a plateau. But, in contrast to fabric anisotropy, its value is higher for polyhedra than for spheres. In other words, the aptitude of the polyhedra packing to develop large force anisotropy is correlated with particle shape rather than with fabric anisotropy (see section 7). The orthoradial force anisotropy $a_{t}$ has a similar behavior except that it takes considerably higher values in the case of polyhedra compared to spheres. In the following section, we study the relationship between the fabric and force anisotropies.

\section{Origins of shear stress}

In this section, we analyze the stresses in the framework of the harmonic approximation of granular microstructure introduced in the last section. Since this representation involves continuous functions of contact orientations, we need to express the stress tensor in integral form. The stress tensor as defined 
in Eq. (3) is an average:

$$
\sigma_{\alpha \beta}=n_{c}\left\langle\ell_{\alpha}^{k} f_{\beta}^{k}\right\rangle_{k},
$$

where $n_{c}=N_{c} / V$ is the number density of contacts, $\ell_{\alpha}^{k}$ is the $\alpha$ component of the branch vector at contact $k$ and $\ell_{\beta}^{k}$ is the $\beta$ component of the force vector at contact $k$. The average is taken over all contacts $k$ in the control volume $V$. To express this mean as an integral, we introduce the joint probability density $P_{\Omega f \ell}(\boldsymbol{n}, \boldsymbol{f}, \ell)$ of the force and branch vectors (Bathurst and Rothenburg [1988], Rothenburg and Bathurst [1989], Ouadfel and Rothenburg [2001]). Then, from Eq. (24), we have

$$
\sigma_{\alpha \beta}=n_{c} \int P_{\Omega f \ell}(\boldsymbol{n}, \boldsymbol{f}, \ell) \ell(\boldsymbol{n}) f_{\beta}(\boldsymbol{n}, \ell) n_{\alpha} d \Omega d \boldsymbol{f} d \ell,
$$

where $d \Omega=\sin \theta d \theta d \phi$.

Equation (25) can be simplified by integrating out the contribution of $\ell$. Assuming that $\boldsymbol{f}$ is independent of $\ell$ (an assumption which is verified with a good approximation), we get

$$
\sigma_{\alpha \beta}=n_{c} \int P_{\Omega f}(\boldsymbol{n}, \boldsymbol{f})\langle\ell\rangle(\boldsymbol{n}) f_{\beta}(\boldsymbol{n}) n_{\alpha} d \Omega d \boldsymbol{f},
$$

where $\langle\ell\rangle P_{\Omega f}=\int P_{\Omega f \ell} d \ell$.

Finally, integration of (26) over force vector yields the following expression for the stress tensor:

$$
\sigma_{\alpha \beta}=n_{c} \int P_{\Omega}(\boldsymbol{n})\langle\ell\rangle(\boldsymbol{n}) n_{\alpha}\left\langle f_{\beta}\right\rangle(\boldsymbol{n}) d \Omega,
$$

where $\langle\boldsymbol{f}\rangle P_{\Omega}=\int P_{\Omega f} d \boldsymbol{f}$. By introducing the average force components $\left\langle f_{n}\right\rangle$ and $\left\langle f_{t}\right\rangle$ in this equation, we get

$$
\sigma_{\alpha \beta}=n_{c} \int P_{\Omega}(\boldsymbol{n})\langle\ell\rangle(\boldsymbol{n})\left\{\left\langle f_{n}\right\rangle(\boldsymbol{n}) n_{\beta}+\left\langle f_{t}\right\rangle(\boldsymbol{n}) t_{\beta}\right\} d \Omega .
$$

This writing of the stress tensor involves the functions previously introduced with the harmonic representation of the fabric (Eqs. (21), (22), (23) and (23)). Inserting these functions in the integral expression (Eq. 281) and given the definitions of mean stress $p$ and stress deviator $q$ in Eq. (5), one gets

$$
\begin{aligned}
p & \simeq n_{c} \ell_{0} f_{0}, \\
\frac{q}{p} & \simeq \frac{2}{5}\left(a+a_{l}+a_{n}+a_{t}\right),
\end{aligned}
$$




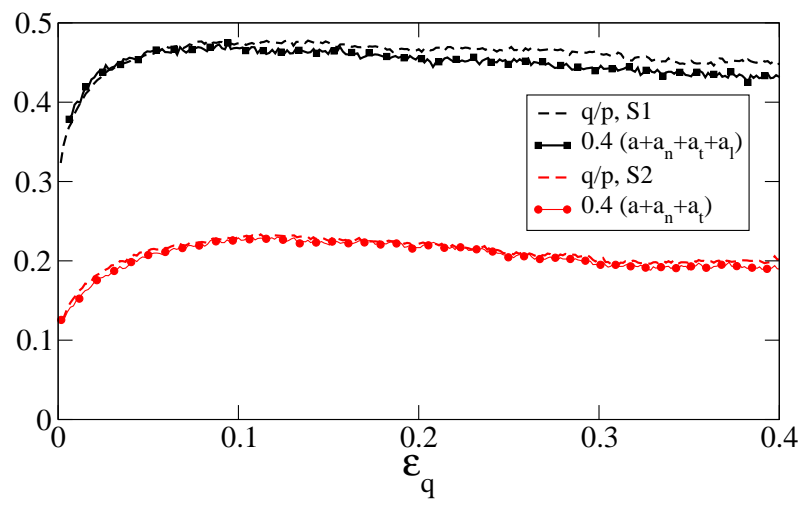

Fig. 13. The normalized shear stress $q / p$ as a function of shear strain $\varepsilon_{q}$ for the packings S1 and S2 both from direct simulation data and theoretical prediction of Eq. (30).

where the cross products $\left(a a_{l}, a a_{n}\right.$ and $\left.a a_{t}\right)$ among the anisotropies have been neglected. Our simulation data are in quantitative agreement with this "stress-force-fabric" relation (a term coined by Rothenburg and Bathurst in (Bathurst and Rothenburg [1988], Rothenburg and Bathurst [1989]) ) both for spheres and polyhedra, as shown in Fig. 13, all along the shear. We note that the theoretical fit would have been less satisfactory for polyhedra if the branch vector length anisotropy $a_{l}$ were omitted from the description.

Equation (30) is interesting as it exhibits the two origins of shear stress in a granular system: 1) the fabric anisotropies $a$ and $a_{l}$, related to the branch vector and 2) the force anisotropies $a_{n}$ and $a_{t}$, related to the contact force. Figure 12 shows that the values of these anisotropy parameters underlying the shear stress depend on the particle shape. In particular, the total force anisotropy $a_{n}+a_{t}$ compared to the total fabric anisotropy $a+a_{l}$ is much higher in the case of polyhedra. In the critical state, we have $a_{n}+a_{t} \simeq 0.88$ and $a+a_{l} \simeq 0.2$ for polyhedra, $a_{n}+a_{t} \simeq 0.26$ and $a+a_{l} \simeq 0.24$ for spheres. The high value of the force anisotropy in the case of polyhedra comes from both radial and orthoradial components whereas in the sphere packing $a_{t} \simeq 0.05$ is much less important than $a_{n} \simeq 0.21$. This suggests that friction is more directly involved in force transmission in the polyhedral packing than in the sphere packing. The strong contribution of force anisotropy to the polyhedra packing is a particle shape effect related to the face-face contacts which carry most strong forces. This point will be analyzed in more detail below.

\section{Force distributions}

In this section, we study the probability density functions (pdf's) $P\left(f_{n}\right)$ for sphere and polyhedra packings. Fig. 14 shows typical maps of normal forces in a portion of both packings in the critical state. The $3 \mathrm{D}$ force chains can 

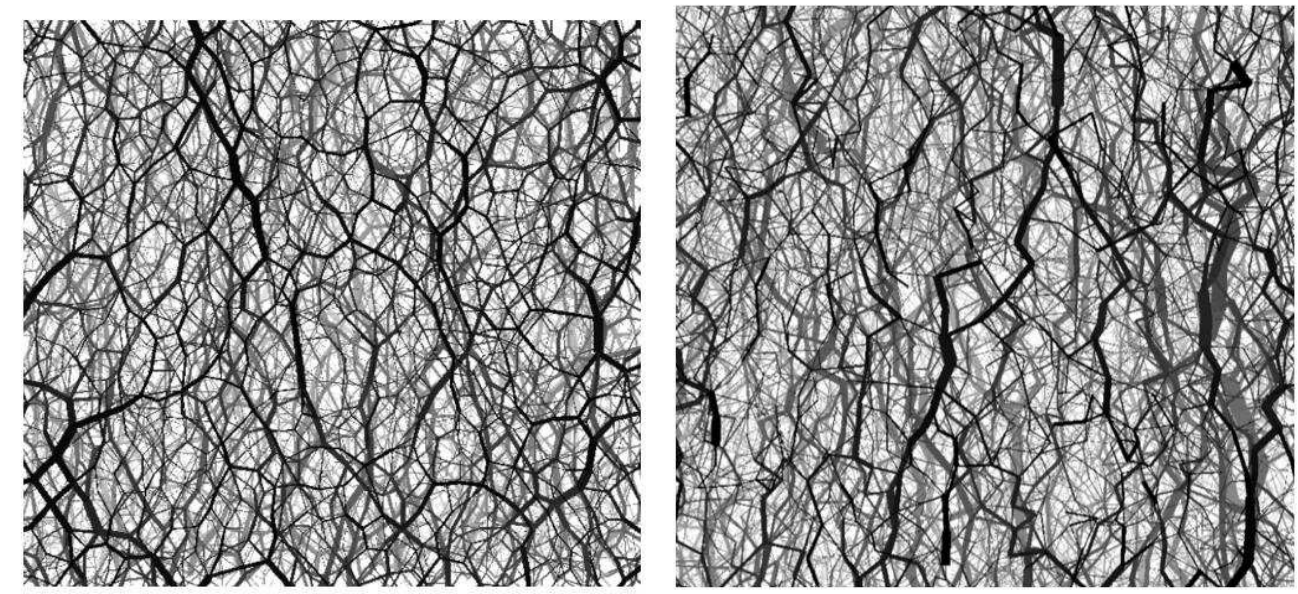

Fig. 14. Force maps in a portion of the packings S1 (right) and S2 (left). The segments are branch vectors with thickness proportional to the normal force, and gray level proportional to the depth of field.
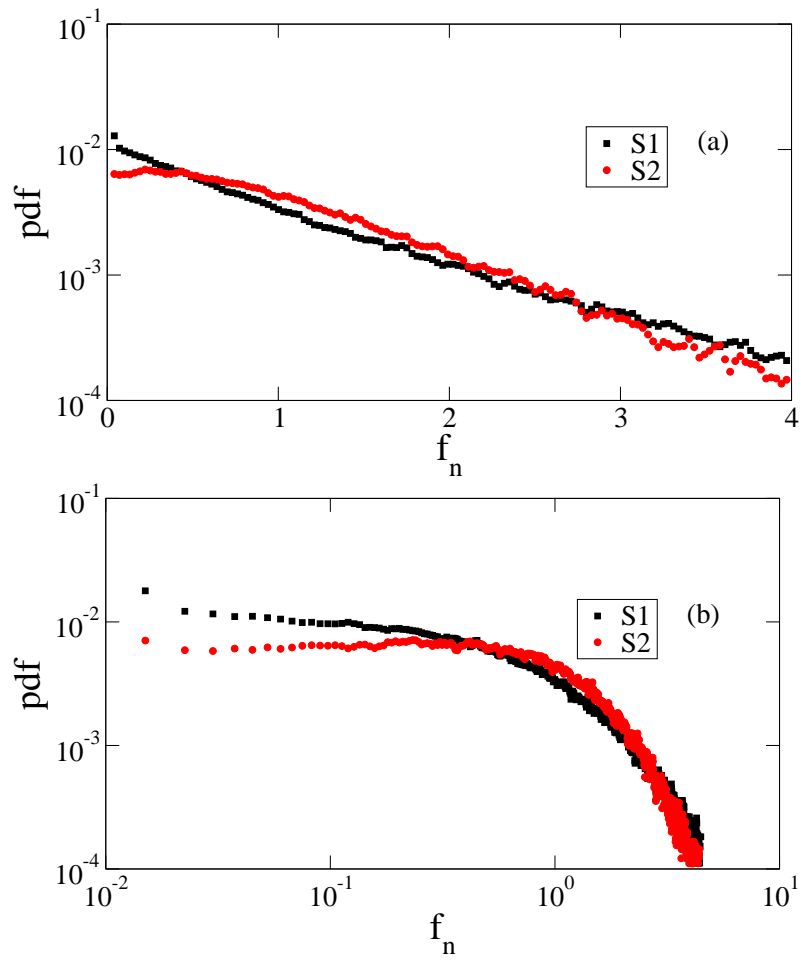

Fig. 15. Probability density functions of normal forces in the packings of spheres and polyhedra.

be observed in both packings, but they seem more tortuous in the case of polyhedra.

The normal force pdf's are shown in Fig. 15 on log-linear and log-log scales at $\varepsilon_{q}=0,3$. In both pdf's, the strong forces, i.e. forces above the mean normal force $\left\langle f_{n}\right\rangle$, fall off exponentially: $P\left(f_{n}\right) \propto e^{-\beta f_{n} /\left\langle f_{n}\right\rangle}$, with $\beta \simeq 0.9$ for S1 and $\beta \simeq 1.1$ for $\mathrm{S} 2$. In contrast, the shapes of the pdf's in the range of 
weak forces $\left(f_{n}<\left\langle f_{n}\right\rangle\right)$ are radically different. In the sphere packing, the pdf slightly bends down as $f_{n} \rightarrow 0$ but does not tend to zero. We observe also a small peak close to the mean force. This is consistent with several other numerical and experimental observations for isotropic packings (Lovol et al. [1999], Bardenhagen et al. [2000], Antony [2001], Silbert et al. [2002], Metzger [2004], Majmudar and Behringer [2005]). In the case of polyhedra, the number of weak forces bends up as the force tends to zero. For both packings, the range of weak forces is well approximated by a power-law distribution :

$$
P\left(f_{n}\right) \propto\left[\frac{f_{n}}{\left\langle f_{n}\right\rangle}\right]^{\alpha}
$$

with $\alpha=-0.24$ for S1 and $\alpha=0.05$ for S2. The divergence of the number of weak forces in $\mathrm{S} 1$ should be attributed to the polyhedral shape of the particles favoring the arching effect an hence a higher fraction of weak forces. The coefficient of friction has a similar effect though to a lesser extent. We find, however, that in both systems the fraction of weak forces $\left(f_{n}<\left\langle f_{n}\right\rangle\right)$ is about $60 \%$.

\section{Contact networks of polyhedral particles}

In the case of the polyhedra packing, it is interesting to investigate the organization of the contact network in terms of simple, double and triple contacts. The respective fractions of these contact types and their contributions to the structural anisotropy and force transmission are the key quantities for understanding the effect of particle shape on the shear strength properties of granular media. In fact, one expects that the triple (face-to-face) contacts play an essential role in force transmission. This feature was observed in the case of polygon packings for side-to-side contacts (Azéma et al. [2007]).

Considering the discrete expression of the stress tensor in Eq. (3) and restricting the summation to each contact type allows us to perform an additive decomposition:

$$
\boldsymbol{\sigma}=\boldsymbol{\sigma}_{s}+\boldsymbol{\sigma}_{d}+\boldsymbol{\sigma}_{t}
$$

where the subscripts $s, d$ and $t$ design the respective contributions of simple, double and triple contacts. The corresponding stress deviators $q_{s}, q_{d}$ and $q_{t}$ are then calculated and normalized by the mean stress $p$. Fig. 16] shows the evolution of partial shear stresses $q_{s} / p, q_{d} / p$ and $q_{t} / p$ as a function of shear strain $\varepsilon_{q}$. The contribution of simple contacts is larger for double and triple contacts. However, the double and triple contacts support together the largest portion of the overall shear stress, i.e. $q_{d}+q_{t}>q_{s}$. 


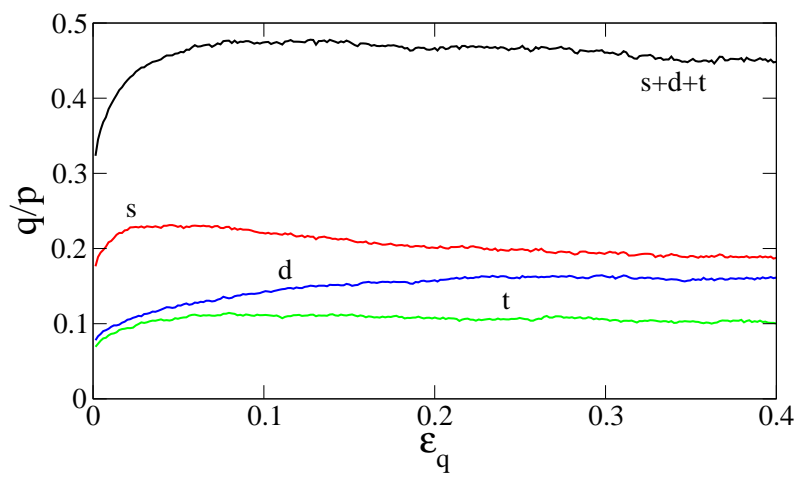

Fig. 16. Evolution of partial shear stresses as a function of shear strain for simple $(\mathrm{s})$, double $(\mathrm{d})$ and triple $(\mathrm{t})$ contacts, as well as the total shear stress $(\mathrm{s}+\mathrm{d}+\mathrm{t})$.

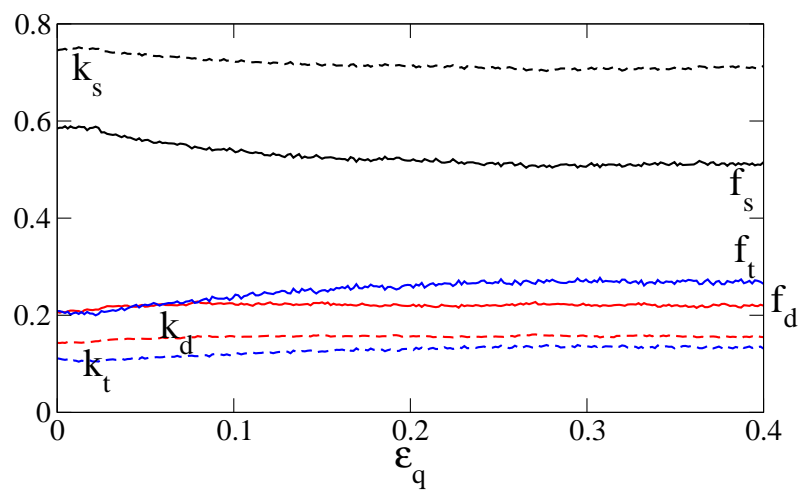

Fig. 17. Proportions $k_{s}, k_{d}$ and $k_{t}$ of simple, double and triple contacts (dashed lines), and the relative average forces $f_{s}, f_{d}$ and $f_{t}$ (full lines) supported by each contact type as a function of shear strain $\varepsilon_{q}$.

The partial shear stress supported by each contact type depends on both the number of its contacts and their mean force. Fig. 17] shows the proportions $k_{s}$, $k_{d}$ and $k_{t}$ of simple, double and triple contacts as a function of shear strain. $k_{s}$ declines during shear from 0.75 to 0.71 whereas $k_{d}$ and $k_{t}$ increase from 0.14 to 0.15 and from 0.11 to 0.14 , respectively. Hence, the critical state is characterized by $k_{s} \simeq 0.7$ et $k_{t} \simeq k_{d} \simeq 0.15$. Fig. 17 also shows the relative mean forces $f_{s}, f_{d}$ and $f_{t}$ defined by

$$
\begin{aligned}
& f_{s}=k_{s}\left\langle f_{n}\right\rangle_{s} /\left\langle f_{n}\right\rangle, \\
& f_{d}=k_{d}\left\langle f_{n}\right\rangle_{d} /\left\langle f_{n}\right\rangle, \\
& f_{t}=k_{t}\left\langle f_{n}\right\rangle_{t} /\left\langle f_{n}\right\rangle,
\end{aligned}
$$

where $\left\langle f_{n}\right\rangle_{s},\left\langle f_{n}\right\rangle_{d}$ and $\left\langle f_{n}\right\rangle_{t}$ correspond to the mean normal forces of simple, double and triple contacts. We see that $f_{s}$ declines slightly with strain but is nearly two times larger than $f_{t}$ and 2.3 times larger than $f_{d}$ in the critical state. We have $f_{s} \simeq f_{d}+f_{t}$. Hence, the lower contribution of triple contacts with respect to shear stress can be attributed to both the low level of the mean force $\left(f_{t}<0.3\right)$ sustained by this class and to their weak number $(<15 \%)$. 


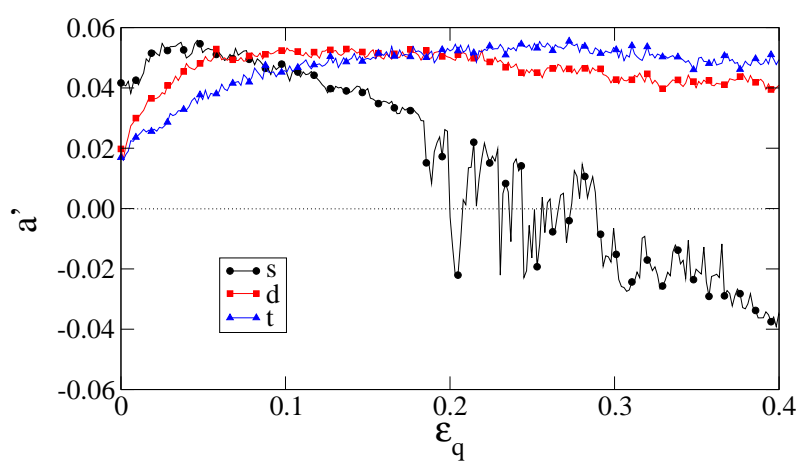

Fig. 18. Evolution of the signed anisotropies $a^{\prime}$ of simple (s), double (d) and triple (t) contacts as a function of shear strain $\varepsilon_{q}$.

Following the same procedure as for the stress tensor, we now perform a similar decomposition of the fabric tensor $\boldsymbol{F}$, defined by Eq. (A.4), into three terms:

$$
\boldsymbol{F}=\boldsymbol{F}_{s}+\boldsymbol{F}_{d}+\boldsymbol{F}_{t}
$$

where $\boldsymbol{F}_{s}, \boldsymbol{F}_{d}$ and $\boldsymbol{F}_{t}$ are the contributions of simple, double and triple contacts. The corresponding anisotropies $a_{s}, a_{d}$ and $a_{t}$ can be extracted, but since the principal directions of these partial fabric tensors are not necessarily identical to those of the overall fabric tensor, we define the "signed" anisotropies by multiplying each partial anisotropy $a_{i}$ by a phase factor $\cos 2\left(\theta_{F}-\theta_{F_{i}}\right)$ :

$$
a_{i}^{\prime}=a_{i} \cos 2\left(\theta_{F}-\theta_{F_{i}}\right) .
$$

Figure 18 shows the evolution of signed anisotropies of the three contact classes. We see that $a_{d}^{\prime}$ and $a_{t}^{\prime}$ increase with shear strain and tend to the limit value $\simeq 0.04$. As to $a_{s}^{\prime}$, we observe an initial increase followed by rapid decrease and change of sign at $\varepsilon_{q} \simeq 0.2$. This evolution means that during shear the branch vectors of simple contacts tend to become increasingly perpendicular to the major principal direction (the direction of compression). A map of contact forces projected along the branch vectors is displayed in Fig. 19 in different colors according to the type of contact. The triple contacts, despite their lower proportion, appear clearly to be correlated in the form of long chains across the packing. These are mostly parallel to the direction of compression. We also observe a large number of weak forces mainly at simple contacts.

The pdf's of normal forces are shown in Fig. 20 separately for simple, double and triple contacts. The three contact types are involved in strong and weak networks. The strong forces have in all cases an exponential behavior but a major difference is observed in the range of weak forces where the proportion 


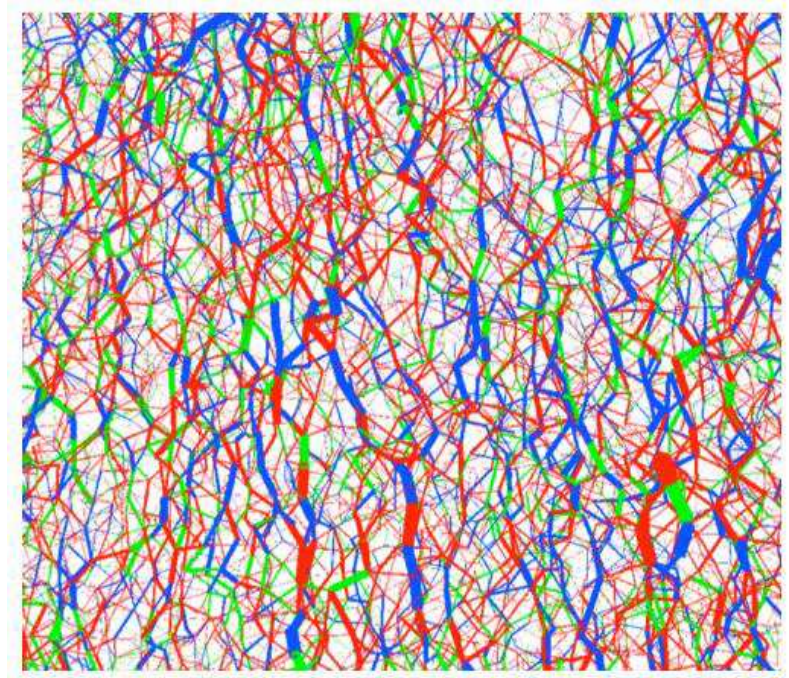

Fig. 19. Map of contact forces projected along branch vectors at $\varepsilon_{q}=0.4$. Line thickness is proportional to the force. The simple, double and triple contacts are in red (dark gray), in green (light gray) and in blue (black).
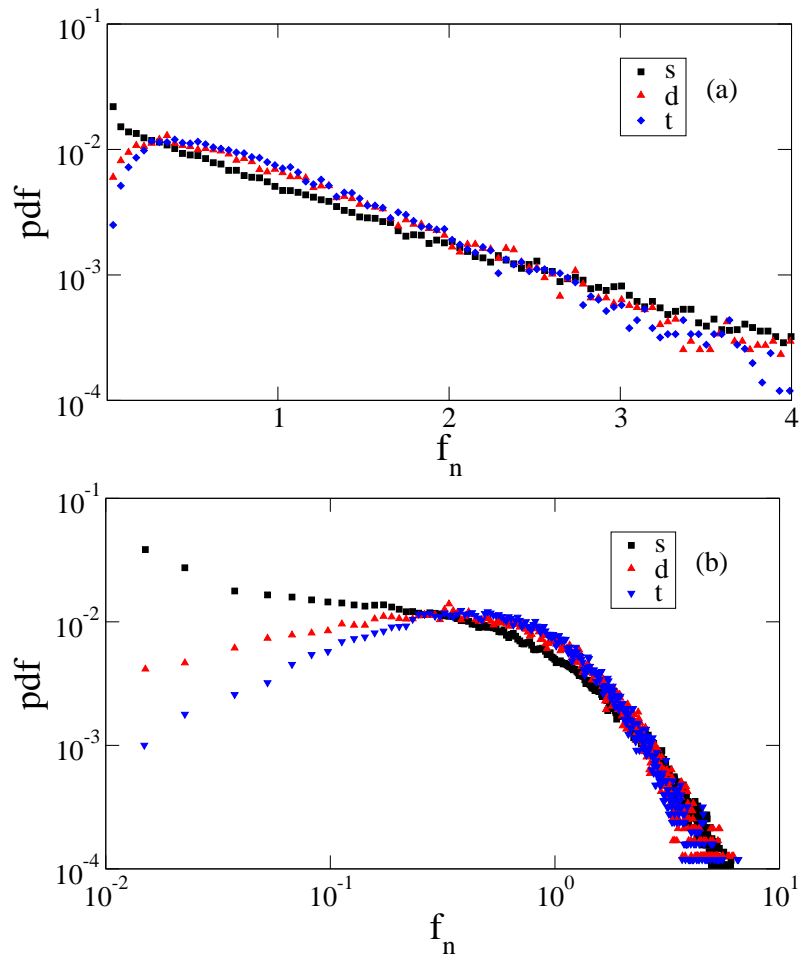

Fig. 20. Probability distribution functions of radial forces at simple (s), double (d) and triple (t) contacts on log-linear (a) and log-log (b) scales.

of simple contacts prevails. This correlation between simple and weak contacts is interesting as it clearly reveals the contrast between simple contacts, on one hand, and double and triple contacts, on the other hand, in the organization of the force network. 


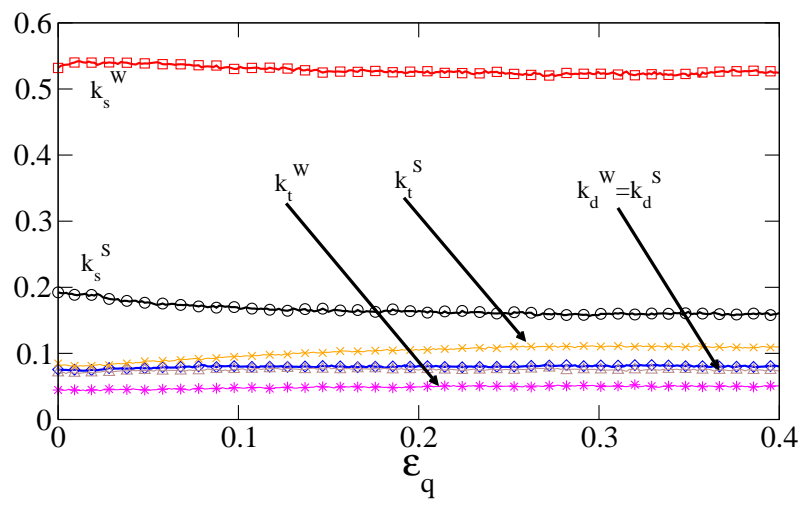

Fig. 21. Proportions $k_{s}^{S}, k_{d}^{S}$ and $k_{t}^{S}$ of simple (s), double (d) and triple (t) contacts in the strong network (S) and the corresponding proportions $k_{s}^{W}, k_{d}^{W}$ and $k_{t}^{W}$ in the weak network (W) as a function of shear strain.

In order to situate the simple, double and triple contacts with respect to the force network, we have plotted in Fig. 21 the proportions $k_{s}^{S}, k_{d}^{S}$ et $k_{t}^{S}$ of the three contact sets in the strong network and the corresponding proportions $k_{s}^{W}, k_{d}^{W}$ et $k_{t}^{W}$ in the weak network as a function of shear strain $\varepsilon_{q}$. It is interesting to note that the proportion of weak simple contacts is quite high $(\simeq 0.55)$. The proportions $k_{d}^{W}$ et $k_{d}^{S}$ of weak and strong double contacts are identical $(\simeq 0.07)$. Finally, we see that most double contacts belong to the strong network $\left(k_{t}^{S} \simeq 2 k_{t}^{W}\right)$.

\section{Conclusion}

In this paper, granular materials composed of irregular polyhedral particles were numerically investigated. Macroscopic and microstructural properties were analyzed by (1) direct comparison with a similar packing composed of spherical particles and (2) characterization of contact networks and force transmission. A novel finding of this work is that the origin of enhanced shear strength in a polyhedra packing compared to a sphere packing lies in force anisotropy induced by particle shape. The fabric anisotropy associated with the network of branch vectors is lower in the polyhedra packing. This finding extends the results of a previous study of pentagonal particles in two dimensions to three dimensions Azéma et al. [2007]. In other words, the force anisotropy, partially underlying shear strength, is mainly controlled by the fabric anisotropy in a sphere packing. This mechanism breaks down to some extent in a packing of polyhedra where force anisotropy results mainly from the "facetted" particle shape.

The face-face contacts were shown to belong mostly to the strong force network. The local equilibrium structures involving face-face and edge-face contacts accommodate force lines that are basically unstable with spherical par- 
ticles. Hence, the term "arching" seems to be more adapted to the description of force patterns in an assembly of polyhedra than in an assembly of spheres. This effect is crucial for the probability density function of normal forces in the range of weak forces that is well approximated by a decreasing power-law in the case of polyhedra.

In this investigation the polyhedra were irregular with a given number of faces, edges and vertices. These shape parameters can now be changed and the resulting packings can be analyzed along the same lines as in the present investigation. Since the face-face contacts seem to play a key role, it would be interesting to consider irregular polyhedra with less faces in number but with larger areas. From a mechanical point of view, there should be little difference between a small face and a vertex. The best shape from the shear strength viewpoint can be obtained with a large number of faces of large area, but these two conditions can not be realized at the same time. It seems thus that an optimal polyhedral shape should exist with a number of faces of not two low areas. The work is under way to elucidate this point and other aspects of the problem concerned with packing structure by systematically changing the particle shape parameters.

We acknowledge assistance by F. Dubois with the LMGC90 platform employed for the simulations, as well as the precious help of V. Richefeu with 3D visualization of forces. This work was funded by the French Railway Society, the SNCF, and the Région Languedoc-Roussillon of France.

\section{A Fabric tensors}

The anisotropies $a, a_{n}, a_{t}$ and $a_{l}$ can be calculated from the tensors $\boldsymbol{F}, \boldsymbol{H}^{(n)}$, $\boldsymbol{H}^{(t)}$ and $\boldsymbol{H}^{(l)}$ defined by (Bathurst and Rothenburg [1988, Rothenburg and Bathurst [1989], Ouadfel and Rothenburg [2001]) :

$$
\begin{aligned}
\boldsymbol{F}_{\alpha \beta} & =\int_{\mathcal{S}} P_{\Omega}(\theta) n_{\alpha} n_{\beta} d \Omega, \\
\boldsymbol{H}_{\alpha \beta}^{(n)} & =\int_{\mathcal{S}}\left\langle f_{n}\right\rangle(\theta) n_{\alpha} n_{\beta} d \Omega, \\
\boldsymbol{H}_{\alpha \beta}^{(t)} & =\int_{\mathcal{S}}\left\langle f_{t}\right\rangle(\theta) n_{\alpha} t_{\beta} d \Omega, \\
\boldsymbol{H}_{\alpha \beta}^{(l)} & =\int_{\mathcal{S}}\langle\ell\rangle(\theta) n_{\alpha} n_{\beta} d \Omega .
\end{aligned}
$$


Using the equations (21), (22),$(23)$ and (23), it is then easy to show that the corresponding anisotropies are :

$$
\begin{aligned}
a & =\frac{5}{2} \frac{F_{3}-F_{1}}{\operatorname{tr} \boldsymbol{F}}, \\
a_{n} & =\frac{5}{2} \frac{H_{3}^{(n)}-H_{1}^{(n)}}{\operatorname{tr} \boldsymbol{H}^{(n)}}, \\
a_{t} & =\frac{5}{2} \frac{H_{3}^{(t)}-H_{1}^{(t)}}{\operatorname{tr} \boldsymbol{H}^{(n)}} \\
a_{l} & =\frac{5}{2} \frac{H_{3}^{(l)}-H_{1}^{(l)}}{\operatorname{tr} \boldsymbol{H}^{(l)}},
\end{aligned}
$$

where $\operatorname{tr} \boldsymbol{H}^{(n)}=\langle f\rangle, \operatorname{tr} \boldsymbol{F}=1$ et $\operatorname{tr} \boldsymbol{H}^{(l)}=\ell_{0}$.

\section{References}

D.W. Airey and D.M. Wood. "The Cambridge true triaxial apparatus", Advanced Triaxial Testing of Soil and Rock. Rebert T. Donaghe, Ronald C. Chaney \& Marshall L. Silver, 1988.

F. Alonso-Marroquin and H. J. Herrmann. Calculation of the incremental stress-strain relation of a polygonal packing. Phys. Rev. E, 66(2):021301-, August 2002.

S. J. Antony. Evolution of force distribution in three-dimensional granular media. Phys Rev E, 63:011302, 2001.

S.J Antony and M.R. Kuhn. Influence of particle shape on granular contact signatures and shear strength: new insights from simulations. International Journal of Solids and Structures, 41(21):5863-5870, October 2004.

E. Azéma, F. Radjai, R. Peyroux, and G. Saussine. Force transmission in a packing of pentagonal particles. Phys. Rev. E, 76:011301, 2007.

S. G. Bardenhagen, J. U. Brackbill, and D. Sulsky. Numerical study of stress distribution in sheared granular material in two dimensions. Phys. Rev. E, 62:3882-3890, 2000.

R. J. Bathurst and L. Rothenburg. Micromechanical aspects of isotropic granular assemblies with linear contact interactions. J. Appl. Mech., 55:17, 1988.

B. Cambou, Ph. Dubujet, and C. Nouguier-Lehon. Anisotropy in granular materials at different scales. Mechanics of Materials, 36(12):1185-1194, December 2004.

S. N. Coppersmith, C.-h. Liu, S. Majumdar, O. Narayan, and T. A. Witten. Model for force fluctuations in bead packs. Phys. Rev. E, 53(5):4673-4685, 1996.

P. A. Cundall and O.D.L. Strack. Discrete numerical model for granular assemblies. geotechnique, 29(1):47-65, 1979. 
P.A. Cundall. Formulation of a three-dimensionnal distinct element modelpart i: a scheme to detect and represent contacts in a system composed of many polyhedral blocks. Int. J. Rock Mech. Min Sci $\&$ Geomech. Abstr., 1988.

F. Dubois and M. Jean. Lmgc90 une plateforme de développement dédiée à la modélisation des problèmes d'intéraction. In Actes du sixième colloque national en calcul des structures - CSMA-AFM-LMS -, volume 1, pages 111-118, 2003.

GDR-MiDi. On dense granular flows. Eur. Phys. J. E, 14:341-365, 2004.

M. Jean and J. J. Moreau. Unilaterality and dry friction in the dynamics of rigid body collections. In Proceedings of Contact Mechanics International Symposium, pages 31-48, Lausanne, Switzerland, 1992. Presses Polytechniques et Universitaires Romandes.

N. P. Kruyt and L. Rothenburg. Micromechanical definition of strain tensor for granular materials. ASME Journal of Applied Mechanics, 118:706-711, 1996.

N. P. Kruyt and L. Rothenburg. Kinematic and static assumptions for homogenization in micromechanics of granular materials. Mechanics of Materials, 36(12):1157-1173, December 2004.

W.L. Lim and G.R. MacDowel. Discrete element modelling of railway ballast discrete element modelling of railway ballast. Granular Matter, 7:19-29, 2005.

C.-h. Liu, S. R. Nagel, D. A. Schecter, S. N. Coppersmith, S. Majumdar, O. Narayan, and T. A. Witten. Force fluctuations in bead packs. Science, 269:513, 1995.

S. Lobo-Guerrero and L. E. Vallejo. Discrete element method analysis of railtrack ballast degradation during cyclic loading. Granular Matter, 8:1952004, 2006.

G. Lovol, K. Maloy, and E. Flekkoy. Force measurments on static granular materials. Phys. Rev. E, 60:5872-5878, 1999.

M. Lu and G.R. McDowel. The importance of modelling ballast particle shape in the discrete element method. Granular Matter, 9:69-80, 2007.

T. S. Majmudar and R. P. Behringer. Contact force measurements and stresseinduced anisotropy in granular materials. Nature, 435:1079-1082, 2005.

J.G.D Morgan E. Markland. The effect of vibration on ballast beds. Geotechnique, 31(3):3,367-386, 1981.

Philip T. Metzger. Granular contact force density of states and entropy in a modified edwards ensemble. Phys. Rev. E, 70(5 Pt 1):051303, Nov 2004.

J.K. Mitchell and K. Soga. Fundamentals of Soil Behavior. Wiley, NY, 2005. J. J. Moreau. Numerical investigation of shear zones in granular materials. In D. E. Wolf and P. Grassberger, editors, Friction, Arching, Contact Dynamics, pages 233-247, Singapore, 1997. World Scientific.

J.J. Moreau. Some numerical methods in multibody dynamics : application to granular. Eur. J. Mech. A/Solids, 13:93-114, 1994.

D. M. Mueth, H. M. Jaeger, and S. R. Nagel. Force distribution in a granular 
medium. Phys. Rev. E., 57(3):3164-3169, 1998.

E.G. Nezami, Y.M.A Hashash, D. Zaho, and J. Ghaboussi. A fast contact detection for 3-d discrete element method. Computers and Geotechnics, 31: 575-587, 2004.

E.G. Nezami, Y.M.A Hashash, D. Zaho, and J. Ghaboussi. Shortest link method for contact detection in discrete element method. Int. J. Numer. Anal. Meth. Geomech., 30:783-801, 2006.

C. Nouguier-Lehon, B. Cambou, and E. Vincens. Influence of particle shape and angularity on the behavior of granular materials: a numerical analysis. Int. J. Numer. Anal. Meth. Geomech, 27:1207-1226, 2003.

H. Ouadfel and L. Rothenburg. 'stress-force-fabric' relationship for assemblies of ellipsoids. Mechanics of Materials, 33(4):201-221, April 2001.

A.A Pena, H. J. Herrmann, A. Lizcano, and F. Alonso-Marroquin. Investigation of the asymptotic states of granular materials using a discrete model of anisotropic particles. In Powders and Grains 2005, pages 697-700. A. A. Balkema, 2005.

A.A. Pena, R. Garcia-Rojo, and H.J. Herrmann. Influence of particle shape on sheared dense granular media. Granular Matter, In Press, 2006a.

A.A. Pena, A. Lizcano, F. Alonso-Marroquin, and H.J. Herrman. Fluctuations at the critical state of a polygonal packing. Int. J. For Numer. Anal. Meth. Geomech., 00:1-12, 2006b.

R. Pérales. Contribution à la modélisation des structures maconnées par approche discrete. Intégration vers une application industrielle. $\mathrm{PhD}$ thesis, Université Montpellier II (en cours), 2007.

F. Radjai and S. Roux. Etats internes des milieux granulaires denses. In $14 e$ Congres Francais de Mécanique. Toulouse, 1999.

F. Radjai and S. Roux. Contact dynamics study of 2d granular media : Critical states and relevant internal variables. In H. Hinrichsen and D. E. Wolf, editors, The Physics of Granular Media, pages 165-186, Weinheim, 2004. Wiley-VCH.

F. Radjai, M. Jean, J.J. Moreau, and S. Roux. Force distributions in dense two dimensional granular systems. Phys. Rev. Letter, 77:274-277, 1996.

F. Radjai, D. E. Wolf, M. Jean, and J.J. Moreau. Bimodal character of stress transmission in granular packings. Phys. Rev. Letter, 80:61-64, 1998.

L. Rothenburg and R. J. Bathurst. Analytical study of induced anisotropy in idealized granular materials. Geotechnique, 39:601-614, 1989.

G. Saussine. Contribution à la modélisation de granulats tridimensionnels : application au ballast. PhD thesis, Université Montpellier II, 2004.

G. Saussine, C. Cholet, P.E. Gautier, F. Dubois, C. Bohatier, and J.J. Moreau. Modelling ballast behaviour under dynamic loading. part1 : A 2d polygonal discrete element method approach. Comput. Methods Appl. Mech. Eng., 195:2841 - 2859, 2006.

L. E. Silbert, G. S. Grest, and J. W. Landry. Statistics of the contact network in frictional and frictionless granular packings. Phys. Rev. E, 66:1-9, 2002.

L. Staron and F. Radjai. Friction versus texture at the approach of a granular 
avalanche. Phys. Rev. E, 72:1-5, 2005.

H. Troadec. Texture locale et plasticité des matériaux granulaires. PhD thesis, Université Montpellier II, 2002.

H. Troadec, F. Radjai, S. Roux, and J.-C. Charmet. Model for granular texture with steric exclusions. Phys. Rev. E, 66:041305, 2002.

D.M. Wood. Soil behaviour and critical state soil mechanics. Cambridge University Press, Cambridge, England, 1990.

Wu and Thompson. The vibration behavior of railway track at high frequencies under multiple preloads and wheel interactions. J Acoust Soc Am, 108(3 Pt 1):1046-1053, Sep 2000. 